Enfermagem Brasil 2018;17(6):662-9

https://doi.org/10.33233/eb.v17i6.2273

\title{
ARTIGO ORIGINAL \\ Perfil das internações de crianças vítimas de queimaduras em um hospital público de Recife
}

Isabel Lopes Pereira da Silva Amaral*, Ana Paula Silva de Brito Rodrigues**, Viviane Maria Pereira de Carvalho Magalhães, M.Sc. ${ }^{* *}$, Sura Wanessa Santos Rocha, D.Sc. ${ }^{* * *}$

**ós-graduada em Saúde Pública com Ênfase em Saúde da Família pela Faculdade de Comunicação e Turismo de Olinda, ${ }^{* * B}$ Bacharela em Enfermagem pela Faculdade de Ciências Humanas de Olinda - FACHO, ${ }^{* * *}$ Enfermeira, Docente da Faculdade de Ciências Humanas de Olinda - FACHO, ${ }^{* * * * D o c e n t e ~ d a ~ U n i v e r s i d a d e ~ d e ~ P e r n a m b u c o ~-~ U P E ~}$

Recebido em 18 de abril de 2018; aceito em 5 de novembro de 2018.

Endereço de correspondência: Isabel Lopes Pereira da Silva Amaral, 1a Travessa Santa Catarina, 25, 53620-373 Igarassu PE, E-mail: isabel20ilps@yahoo.com.br; Ana Paula Silva de Brito Rodrigues: paulabrito784@gmail.com; Viviane Maria Pereira de Carvalho Magalhães: viviclistenis@yahoo.com.br; Sura Wanessa Santos Rocha: surawanessa@gmail.com

\section{Resumo}

Objetivo: Verificar o perfil das internações de crianças vítimas de queimaduras em um hospital de referência no atendimento a pacientes queimados de Recife. Métodos: Estudo retrospectivo, descritivo e quantitativo por meio da análise de prontuários de pacientes pediátricos internados entre 2014 e 2015 no setor infantil de queimados do hospital. Os dados foram coletados com apoio de um formulário elaborado e preenchido pelas pesquisadoras, contendo as seguintes variáveis: idade, sexo, classificação da queimadura, porcentagem SCQ, agente causador e tempo de internação. A coleta foi realizada no período de setembro a novembro de 2017. Resultados: A faixa etária predominante foi de 0 a 2 anos, sendo o sexo masculino mais acometido, o tempo de internação variou de 1 a 10 dias, o principal agente causador foram os líquidos quentes, a classificação mais frequente de queimadura foi a de $2^{\circ}$ grau, o percentual de superfície corporal queimada predominante foi de 1 a $8 \%$. Conclusão: $O$ estudo evidencia a necessidade de desenvolvimento de estratégias de educação em saúde para redução dos números alarmantes de crianças queimadas.

Palavras-chave: cuidados de enfermagem, unidade de queimados, crianças, prevenção de acidentes.

\section{Abstract \\ Profile of children's victims of burns in a public hospital of Recife}

Objective: To verify the hospitalization profile of burn victims in a referral hospital for patients burned from Recife. Methods: Retrospective, descriptive and quantitative study by analyzing medical records of hospitalized pediatric patients between 2014 and 2015 in the hospital burned children's ward. The data were collected with the help of a form elaborated and filled out by the researchers, containing the following variables: age, gender, burn classification, percent of total body surface area burned (TBSA), causative agent and length of hospital stay. The collection was carried out from September to November 2017. Results: The predominant age group was 0 to 2 years old, the male gender was more affected, the hospitalization time ranged from 1 to 10 days, the main causative agent was hot liquids, the most frequent burn classification was the 2nd degree, the predominant percentage of burned body surface area was 1 to $8 \%$. Conclusion: The study highlights the need to develop health education strategies to reduce the alarming numbers of burned children.

Key-words: nursing care, burns, child, accidents. 


\section{Resumen \\ Perfil de las internaciones de niños víctimas de quemaduras en un hospital público de Recife}

Objetivo: Verificar el perfil de las internaciones de niños víctimas de quemaduras en un hospital de referencia en la atención a pacientes quemados de Recife. Métodos: Estudio retrospectivo, descriptivo y cuantitativo por medio del análisis de prontuarios de pacientes pediátricos internados entre 2014 y 2015 en el sector infantil de quemados del hospital. Los datos fueron recolectados con apoyo de un formulario elaborado y llenado por las investigadoras, conteniendo las siguientes variables: edad, sexo, clasificación de la quemadura, porcentaje de Superficie Corporal Quemada (SCQ), agente causante y tiempo de internación. La recolección fue realizada en el período de septiembre a noviembre de 2017. Resultados: El grupo de edad predominante fue de 0 a 2 años, siendo el sexo masculino más acometido, el tiempo de internación varió de 1 a 10 días, el principal agente causante fueron los líquidos calientes, la clasificación más frecuente de quemadura fue la de $2^{\circ}$, el porcentaje de superficie corporal quemada predominante fue de 1 al $8 \%$. Conclusión: El estudio evidencia la necesidad de desarrollar estrategias de educación en salud para reducir los números alarmantes de niños quemados.

Palabras-clave: atención de enfermería, quemaduras, niño, accidentes.

Introdução

O acidente não ocorre ao acaso, há um conjunto de fatores associados que estão ligados à vítima suscetível, ao agente lesivo e ao ambiente inseguro. Embora possam ocorrer em outros ambientes, é no domicílio que ocorre a maior parte dos acidentes [1].

Dentre os acidentes, destacam-se as queimaduras que são feridas traumáticas causadas na maioria das vezes por agentes térmicos, químicos, elétricos ou radioativos. Atuam nos tecidos de revestimento do corpo humano, determinando destruição parcial ou total da pele e seus anexos, podendo atingir camadas mais profundas, como o tecido celular subcutâneo, músculos, tendões e ossos [2].

Segundo a Organização Mundial de Saúde (OMS) a maioria das queimaduras ocorre no ambiente doméstico pelo contato direto com líquidos quentes, chamas ou fonte de calor compostos por objetos como ferro de passar, panelas, fornos entre outros. A maior ocorrência é com crianças, pois são incapazes de identificar o que pode ser perigoso e trazer dano à saúde. A negligência dos pais ou responsáveis pode ser um fator agravante [3].

Queimaduras ocupam 4ㅇ lugar num ranking de mortalidade por acidentes, pelo menos 1 milhão de acidentes desse tipo aconteçam por ano no Brasil, sendo 300 mil em crianças. Acidentes por queimaduras são responsáveis pelo $2^{\text {o }}$ lugar em relação ao número de hospitalizações [4]. São gastos pelo Sistema Único de Saúde (SUS) aproximadamente 55 milhões de reais por ano com o tratamento de pacientes vítimas de queimaduras [5]. Cerca de $75 \%$ das crianças vítimas de queimaduras terá alguma sequela, e precisará de cuidados médicos, cirúrgicos, psicológicos e intervenção para sua reabilitação [6].

Crianças vítimas de queimaduras estão expostas a inúmeras complicações graves, que se dão através das feridas e alterações sistêmicas, pois seu corpo irá agir para repor todos os danos causados pela lesão tecidual. Há fatores importantes para a classificação da gravidade da lesão, como o local da ferida, a idade, o estado geral da criança, o agente causador e o comprometimento respiratório [7].

$\mathrm{Na}$ Estratégia de Saúde da Família, um dos instrumentos utilizados para acompanhar crianças é o programa de puericultura, que tem como propósito acompanhar o crescimento e desenvolvimento da criança. As consultas de puericultura são realizadas pelo enfermeiro e dentre as atribuições pode-se destacar a função de alertar os pais sobre a prevenção dos diversos acidentes que podem ocorrer na infância [8].

O enfermeiro também tem a oportunidade de realizar ações de orientação educacional, por estar inserido na comunidade e ter o privilégio do contato direto com as famílias. Nas visitas domiciliares ele contribui para minimização dos acidentes por queimaduras, orientando os pais sobre as precauções a serem tomadas principalmente na cozinha [9].

Diante do que se expõe, surge a questão norteadora do estudo: Qual o perfil das internações de crianças vítimas de queimaduras? O presente estudo teve o objetivo de verificar o perfil das internações de crianças vítimas de queimaduras em um hospital de referência em Recife/PE, com o intuito de fornecer subsídios científicos aos profissionais de saúde para orientar as ações de prevenção e consequentemente diminuir gastos públicos. 
Trata-se de um estudo retrospectivo, descritivo e quantitativo por meio da verificação de prontuários de pacientes pediátricos internados na unidade de tratamento de queimados de um hospital de referência no atendimento a pacientes vítimas de queimaduras em Recife. A população foi composta pelos prontuários de crianças vítimas de queimaduras internadas no setor infantil de queimados, tendo como amostra prontuários de crianças com idade de 0 a 11 anos 11 meses e 29 dias, de ambos os sexos, internados no período de 2014 a 2015 . Foram excluídos prontuários que não continham as informações de interesse do estudo.

A coleta de dados foi realizada nos meses de setembro a novembro de 2017 utilizando um formulário elaborado e preenchido pelas pesquisadoras, contendo as seguintes variáveis: idade, sexo, classificação da queimadura, porcentagem SCQ, agente causador e tempo de internação. O início da coleta se deu após aprovação do Comitê de Ética em Pesquisa do Hospital da Restauração em Recife/PE conforme CAAE 73520217.4.0000.5198. Este trabalho atendeu à Resolução 466/12 do Conselho Nacional de Saúde referente à pesquisa com seres humanos.

Para organização dos dados, utilizou-se planilha do programa Microsoft Excel versão 2010. A análise foi dividida em duas partes: análise descritiva, onde se calculou estatísticas descritivas simples, como média, mediana, desvio padrão, mínimo e máximo, e análise inferencial, através de testes estatísticos. Todas as análises foram realizadas utilizando o software estatístico R. Os testes estatísticos foram aplicados para determinar quais variáveis possuem relação estatisticamente significativa. Para verificar associação entre variáveis qualitativas foram aplicados os testes de Qui-quadrado. Para estabelecer a existência de relação entre variáveis qualitativas e quantitativas foram ajustados modelos ANOVA e aplicados testes $\mathrm{F}$ a fim de determinar se a média das variáveis quantitativas era igual em todas as categorias das variáveis qualitativas. Por fim, para determinar quais variáveis quantitativas estavam correlacionadas foi estudada a Correlação Linear de Pearson. Utilizou-se nesse estudo uma significância estatística de $5 \%$.

Resultados e discussão

De acordo com os dados obtidos sobre a faixa etária de crianças internadas por queimaduras nos anos de 2014 e 2015, a mais acometida foi a de 0 a 2 anos com percentual de $63 \%$, e a menos acometida foi de 7 a 11 anos com percentual de $14 \%$ (Tabela I). Uma pesquisa epidemiológica e documental realizada em um hospital público estadual de referência para queimados na capital paraibana em 2010 se alinha ao presente estudo no que diz respeito ao intervalo de idades de crianças mais acometidas por queimaduras, pois obteve o resultado de $37 \%$ de crianças de 0 a 2 anos internadas devido a acidentes por queimadura, o que significou a maior parte de sua amostra [9].

Outra pesquisa que também se alinha, foi realizada no município de Aracaju/SE em 2016. Trata-se de um estudo retrospectivo realizado a partir dos dados do sistema de registro de cirurgia plástica da unidade de tratamento de queimados de um hospital de urgências em Sergipe. Crianças com idade de 0 a 2 anos foram responsáveis por $84,39 \%$ das internações [10].

Uma das justificativas para alta prevalência em crianças nesse intervalo de idade explica-se pelo fato do desenvolvimento nessa fase ser marcado pelo entusiasmo e curiosidade em explorar o meio onde vive [9].

Os fatores de risco e de vulnerabilidade para acidentes em crianças podem ser divididos em: intrapessoais, que estão relacionados à idade, sexo e comportamento; interpessoais, que são relacionados aos cuidados pela família e ao ambiente doméstico; institucionais que podem estar ligados à comunidade e escola; e fatores culturais, são aqueles relacionados à sociedade. As crianças menores de 2 anos além de quererem explorar o meio, estão sujeitas a riscos impostos por terceiros, ou seja, grande parte dos acidentes que acontecem com essas crianças está relacionada com a negligência dos pais e cuidadores, pois aos dois anos a criança não tem consciência do que pode trazer danos à saúde [11]. 
Tabela I - Distribuição de crianças internadas por queimaduras de acordo com a faixa etária. Recife, 2014-2015.

\begin{tabular}{lllll}
\hline $\begin{array}{l}\text { Distribuição de acordo } \\
\text { com a faixa etária }\end{array}$ & $\mathbf{N}$ & $\%$ & $\begin{array}{l}\text { Média } \\
\text { (DP) }\end{array}$ & $\begin{array}{l}\text { Mediana } \\
\text { (Min-Máx) }\end{array}$ \\
\hline 0 a 2 anos & 355 & 63 & - & - \\
\hline 3 a 6 anos & 130 & 23 & - & - \\
\hline 7 a 11 anos & 80 & 14 & - & - \\
\hline Total & 565 & 100 & 2,02 & 1 \\
& & & 2,92 & $(0,05-11)$ \\
\hline
\end{tabular}

Fonte: Dados coletados na pesquisa.

No que diz respeito à distribuição de crianças de acordo com o sexo, o masculino teve percentual de $57 \%$ da amostra e o feminino com $43 \%$. O presente estudo apesar de não constatar um forte impacto na diferença dos sexos concorda com uma pesquisa retrospectiva e descritiva realizada a partir da coleta em prontuários de crianças internadas no Centro de tratamento de queimados de um hospital em Londrina/PR no ano de 2014, que revela o predomínio do sexo masculino com percentual de 69,4\% [12].

Outro estudo que se alinha a essa pesquisa foi realizado com crianças de 0 a 14 anos internadas em um Pronto Socorro para queimaduras em Goiânia/GO. Trata-se de um estudo documental no período de 2005 a 2007, ao analisar o sexo acometido, obteve-se percentual de $64,1 \%$ em crianças do sexo masculino [13].

O predomínio de queimaduras em crianças do sexo masculino pode estar relacionado às diferenças de comportamento de cada sexo, geralmente os meninos brincam com mais liberdade e se expõem a situações de risco, quando as meninas geralmente são mais cautelosas e mais vigiadas [14].

Em relação ao tempo em que a criança queimada ficou internada, a pesquisa revela que a maioria permaneceu de 1 a 10 dias com percentual de $76 \%$, apenas $20 \%$ permaneceu de 11 a 20 dias e acima de 21 dias os resultados foram irrisórios (Tabela II). Em um estudo documental realizado com crianças queimadas no município de João Pessoa/PB no ano de 2010, a média de dias de internação foi de 5,87 dias [9]. No entanto, o estudo realizado em Londrina/PR em 2012 revela que o tempo médio de internação foi de 14,8 dias [15].

Uma pesquisa que se alinha aos resultados obtidos no presente estudo foi realizada com prontuários de crianças internadas no período de 2002 a 2012 em uma unidade de tratamento de queimados de um hospital em Catanduva/SP. A maioria dos pacientes $(61,5 \%)$ permaneceu no período de 1 a 9 dias. A variação de dias de internação pode estar associada à condição clínica de cada paciente bem como procedimentos e medicamentos utilizados [16].

Tabela II - Distribuição de crianças internadas por queimaduras de acordo com o tempo de internação. Recife 2014-2015.

\begin{tabular}{lllll}
\hline $\begin{array}{l}\text { Distribuição de acordo com } \\
\text { o tempo de internação }\end{array}$ & $\mathbf{N}$ & $\%$ & $\begin{array}{l}\text { Média } \\
\text { (DP) }\end{array}$ & $\begin{array}{l}\text { Mediana } \\
\text { (Min - Máx) }\end{array}$ \\
\hline 1 a 10 dias & 430 & 76 & - & - \\
\hline 11 a 20 dias & 112 & 20 & - & - \\
\hline 21 a 30 dias & 19 & 3 & - & - \\
\hline 31 a 40 dias & 2 & 0,5 & - & - \\
\hline 41 a 50 dias & 2 & 0,5 & - & - \\
\hline Total & 565 & 100 & 7,78 & 6 \\
& & & 6,13 & $(1-50)$
\end{tabular}

Fonte: Dados coletados na pesquisa.

A tabela III demonstra os resultados da distribuição das crianças de acordo com o agente causador da queimadura. $O$ agente que ocupa o primeiro lugar com percentual de $81 \%$ é a escaldadura e o agente menos frequente foram os fogos de artifício com percentual de $1 \%$. A escaldadura também foi a causa mais prevalente de acidentes por queimaduras em todas as faixas etárias de um estudo realizado na cidade de São Paulo/SP em 2010, tendo discreto aumento em crianças de até 5 anos de idade [17]. Um estudo realizado em 2009, na cidade de Goiânia/GO, dividiu as crianças em dois grupos: grupo I, de 0 a 4 anos e grupo II, de 5 a 14 anos. No grupo I, o principal agente causador foi a escaldadura com percentual de 67,4\% [13].

No entanto, uma pesquisa, realizada em Londrina/PR no ano de 2014, revelou que 0 principal agente causador de queimaduras em crianças de 7 a 12 anos foram chamas e 
líquidos inflamáveis, evidenciando que acidentes por escaldadura acometem mais crianças na primeira infância [12].

A inquietude e desconhecimento das crianças bem como aglomeração na cozinha, junto à mãe, principalmente durante a preparação dos alimentos, justifica a maioria dos estudos apontar a escaldadura como principal agente causador de queimaduras. Há uma condição conhecida como "Síndrome da chaleira quente", que resulta da ação da criança em deslocar do fogão um recipiente com água fervente ou outros líquidos quentes. Atrelado a isso se pode suscitar a negligência dos pais como fator agravante, tendo em vista que a cozinha não é o local mais apropriado para permanência de crianças [9].

Tabela III - Distribuição de crianças internadas por queimaduras de acordo com o agente causador. Recife 2014 - 2015.

\begin{tabular}{lll}
\hline Distribuição de acordo com o agente causador da queimadura & $\mathbf{N}$ & $\%$ \\
\hline Escaldadura & 455 & 81 \\
\hline Superfície aquecida & 35 & 7 \\
\hline Choque elétrico & 33 & 6 \\
\hline Chamas & 24 & 4 \\
\hline Líquidos inflamáveis & 10 & 1 \\
\hline Fogos de artifício & 8 & 1 \\
\hline Total & 565 & 100 \\
\hline
\end{tabular}

Fonte: Dados coletados na pesquisa.

De acordo com os dados desta pesquisa, as crianças foram mais acometidas por queimaduras de $2^{\circ}$ grau, apresentando percentual de $88 \%$. As queimaduras de $1 \%$ impactaram $7 \%$ e apenas $5 \%$ foram acometidos por queimadura de 30 grau. O presente estudo concorda com dados revelados em uma pesquisa realizada no ano de 2016 em Aracaju/SE, que apresentou mais da metade dos casos $(85,8 \%)$ de crianças com queimaduras de $2^{\circ}$ grau [10]. Quanto ao grau da queimadura, outra pesquisa se alinha ao presente estudo, quando revela que dois terços de sua amostra sofreram queimaduras de $1^{\circ}$ e $2^{\circ}$ graus [16].

Outro trabalho que também apresentou resultado semelhante foi realizado no Hospital Universitário de Londrina. Crianças apresentam a pele mais sensível, por isso as queimaduras superficiais tendem a se transformar em queimaduras mais profundas. A profundidade da queimadura está diretamente relacionada ao tempo de exposição e a temperatura que a criança foi submetida [15].

No que diz respeito à Superfície Corporal Queimada (SCQ), a maioria das crianças tiveram de 1 a $8 \%$, atingindo um percentual de $75 \%$ da amostra. Crianças acometidas por queimaduras que comprometessem de 9 a $16 \%$ da SCQ representaram $20 \%$ e poucas foram as crianças com mais de $17 \%$ de extensão do corpo queimado. A média geral para todos os pacientes foi $6,95 \%$ (Tabela IV). Os resultados obtidos discordam de dados apresentados em pesquisa com crianças internadas em uma unidade de tratamento de queimados no interior de São Paulo, a SCQ foi de aproximadamente $18 \%$ para a maioria dos investigados [16]. Vale ressaltar que as crianças têm maior SCQ se comparada com um adulto vítima de queimadura exposto ao mesmo agente pelo mesmo tempo de duração [13].

O paciente pode ser classificado como pequeno queimado, quando a SCQ é menor que $10 \%$, médio queimado quando a SQC é entre $10 \%$ e $20 \%$ e grande queimado se a lesão compromete mais de $20 \%$. A tabela de Lund-Browder é mais confiável para cálculos em crianças [18].

Tabela IV - Distribuição de crianças internadas por queimaduras de acordo com a SCQ. Recife 2014-2015.

\begin{tabular}{lllll}
\hline $\begin{array}{l}\text { Distribuição de acordo com a } \\
\text { porcentagem da SQC }\end{array}$ & $\mathbf{N}$ & $\%$ & $\begin{array}{l}\text { Média } \\
\text { (DP) }\end{array}$ & $\begin{array}{l}\text { Mediana } \\
\text { (Min - Máx) }\end{array}$ \\
\hline 1 a $8 \%$ & 427 & 75 & - & - \\
\hline 9 a $16 \%$ & 112 & 20 & - & - \\
\hline 17 a $24 \%$ & 17 & 3 & - & - \\
\hline 25 a 32\% & 7 & 1,5 & - & - \\
\hline 33 a $100 \%$ & 2 & 0,5 & - & - \\
\hline Total & 565 & 100 & $6,956,32$ & $5(1-100)$ \\
\hline
\end{tabular}

Fonte: Dados coletados na pesquisa. 
$\mathrm{Na}$ análise inferencial realizada através de testes estatísticos, foi possível identificar algumas relações estatisticamente significativas. Apresentamos na Tabela $V$ os resultados dos testes de Qui-quadrado, Testes F e o teste de Correlação Linear de Pearson.

Tabela V - Correlação entre as variáveis.

Resultados dos testes de qui-quadrado

\begin{tabular}{llll}
\hline Variável 1 & Variável 2 & Estatística & p-valor \\
\hline Sexo & Agente causador & 10.71 & 0.05749 \\
\hline Sexo & Grau & 0.86 & 0.65119 \\
\hline Agente Causador & Grau & 130.70 & $<0.001^{*}$ \\
\hline Resultados dos testes F & & & \\
\hline Variável 1 & Variável 2 & $\mathbf{F}$ & p-valor \\
\hline Sexo & Idade & 3.11 & 0.07856 \\
\hline Sexo & Tempo de internação & 0.27 & 0.60242 \\
\hline Sexo & SCQ & 0.98 & 0.32234 \\
\hline Agente causador & Idade & 17.39 & $<0.0001^{*}$ \\
\hline Agente causador & Tempo de internação & 9.43 & $<0.0001^{*}$ \\
\hline Agente causador & SCQ & 7.46 & $<0.0001^{*}$ \\
\hline Grau & Idade & 6.40 & $0.00179^{*}$ \\
\hline Grau & Tempo de Internação & 40.90 & $<0.0001^{*}$ \\
\hline Grau & SCQ & 13.09 & $<0.0001^{*}$ \\
\hline $\begin{array}{l}\text { Resultados dos testes de } \\
\text { correlação }\end{array}$ & & & \\
\hline Variável 1 & Variável 2 & Correlação & p-valor \\
\hline Idade & Tempo de Internação & 0.073 & 0.08434 \\
\hline Idade & SCQ & -0.052 & 0.21753 \\
\hline Tempo de Internação & SCQ & 0.442 & $<0.0001^{*}$ \\
\hline${ }^{*}$ Significância de 5\%. & & &
\end{tabular}

No tocante à relação estatística de agente causador e grau, o estudo evidenciou que crianças que sofreram queimaduras por escaldadura tiveram maior inclinação para terem queimaduras de $2^{\circ}$ grau. Das 455 crianças que foram queimadas por escaldadura, 426 tiveram queimaduras de $2^{\circ}$ grau. Uma pesquisa, realizada com prontuários de crianças atendidas em um hospital de referência no atendimento a pacientes queimados em Salvador, afirma que em sua amostra foi possível identificar que o agente causador da queimadura está diretamente ligado com a profundidade da lesão, e seus resultados se equiparam ao do presente estudo [19].

A relação entre agente causador e idade demonstrou que crianças que sofreram queimaduras por fogos de artifício tinham mais idade, a média foi de 9,38 anos, enquanto que por escaldadura eram mais novas, a média foi de 2,64 anos. Uma pesquisa documental realizada no estado de São Paulo confirma que crianças de 0 a 3 anos tendem a ser mais acometidas por escaldadura, o que confirma os dados do presente estudo. Porém essa mesma pesquisa em São Paulo mostra que os líquidos inflamáveis são responsáveis pelas queimaduras em crianças de mais de 9 anos, nesta presente pesquisa são os fogos de artifício [20].

Na relação agente causador e tempo de internação foi possível verificar que crianças internadas por queimaduras causadas por líquidos inflamáveis permanecem internadas uma média de 16 dias, enquanto que queimaduras por superfície aquecida permanecem menos tempo com uma média de 4,74 dias. E na relação do agente com a SCQ, identificou-se uma média de $11,55 \%$ de superfície corporal queimada para os pacientes queimados com os líquidos inflamáveis e 11,56\% para chamas. A extensão da queimadura foi em média de 7,09\% para as vítimas por escaldadura e $3,66 \%$ para crianças que se queimaram com superfícies aquecidas.

Além de os líquidos inflamáveis serem responsáveis pela maior média de tempo de internação, eles também provocaram queimaduras mais extensas ficando atrás apenas das chamas por $0,01 \%$. Embora acidentes com líquidos inflamáveis sejam mais comuns em adultos, pelo fato de usarem, por exemplo, o álcool para limpeza e acender churrasqueiras, as crianças 
por se tornarem mais independentes, passam a frequentar ambiente extradomiciliar, fazendo com que as queimaduras por combustão e explosão ocorram com frequência, além disso, por vezes materiais inflamáveis ficam em fácil acesso dentro do próprio domicílio também [21].

Houve significância estatística entre a variável tempo de internação e superfície corporal queimada. As crianças com maiores porcentagens de SCQ ficaram mais tempo internadas. Um estudo realizado em Londrina no ano de 2016 evidenciou um menor tempo de internação comparado a outra pesquisa realizada no mesmo local em um período anterior. Isso se deu, pois a média de SCQ também era menor [22]. O grau de queimadura assim como a extensão também tem relação significativa com o tempo de internação. Crianças acometidas por queimaduras de 3 o grau permaneceram em média 15,57 dias internadas, enquanto que as de $2^{\circ}$ grau ficaram 7,69 dias e de $1^{\circ}$ grau uma média de 3,24 dias. Então se conclui que quanto maior o grau e/ou a extensão da queimadura maior será o tempo de internação.

A classificação da queimadura quanto ao grau e a SCQ também são estatisticamente relacionados. Quanto maior o grau da queimadura, maior também foi a extensão nos pacientes deste estudo. Crianças com queimaduras de 3 o grau tiveram em média $11,13 \%$ de extensão de queimadura, queimaduras de $2^{\circ}$ grau $6,98 \%$ e queimaduras de $1^{\circ}$ grau $3,58 \%$ de SCQ.

A idade das crianças apresentou significância estatística com o grau de queimadura. Lesões por queimaduras de $3^{\circ}$ grau acometeram crianças de uma média de 4,53 anos, as de $2^{\circ}$ grau crianças de com 2,86 anos em média e 1ํgrau com 3,86 anos. As idades são próximas, mas se percebe que as mais novas sofrem queimaduras de $2^{\circ}$ grau. Faz sentido já que as mais novas sofrem mais com escaldadura e esse agente causador acomete mais a faixa dos dois anos de idade.

\section{Conclusão}

Os dados coletados são compatíveis com resultados de outros estudos quando mostram que há maior prevalência em crianças do sexo masculino, com idade de zero a dois anos, e o principal agente são líquidos quentes e mais da metade têm queimaduras de segundo grau. Com isso, revela-se a necessidade de estratégias voltadas para prevenção de queimaduras em crianças.

O enfermeiro na Atenção Básica tem um papel crucial para alertar os pais sobre a prevenção de acidentes, esse ponto é um dos pilares das consultas de puericultura. Deve-se reforçar sobre os perigos das crianças permanecerem na cozinha, o perigo de produtos inflamáveis ficarem com fácil acesso e as tomadas sem proteção que podem causar queimaduras por choque elétrico.

Além do profissional de enfermagem, se faz necessária a mobilização das equipes de atenção básica para visitação em escolas, realizando palestras educativas para crianças com mais idade, com o intuito de alertá-los sobre os perigos dos fogos de artifício, não usar fósforos e nunca usar celulares ou outros aparelhos quando estiver carregando, além de outras medidas de proteção. A educação em saúde sempre será relevante para prevenção de acidentes.

1. Vasconcelos JMB, Souza ATO, França JRFS. Acidentes na infância no ambiente domiciliar: proposta educativa de enfermagem. Congresso Brasileiro de Enfermagem do Trabalho 3237-1/4; 2009.

2. Sociedade Brasileira de Queimaduras. Queimaduras [Internet]. Goiânia/GO; 2015. [citado 2017 Set 8]. Disponível em: http://sbqueimaduras.org.br/queimaduras-conceitoe-causas/.

3. Brito MEM, Damasceno AKC, Pinheiro PNC, Vieira LJES. A cultura no cuidado familiar à criança vítima de queimaduras. Rev Eletr Enferm 2010;12(2):321-5.

4. Sociedade Brasileira de Queimaduras. Queimaduras são a quarta maior causa de morte entre as crianças [Internet]. Goiânia, GO; 2014. [citado 2017 Set 8]. Disponível em: http://sbqueimaduras.org.br/queimaduras-sao-a-quarta-maior-causa-de-morteentre-as-criancas/.

5. Costa GOP, Silva JA, Santos AG. Perfil clínico e epidemiológico das queimaduras: evidências para o cuidado de enfermagem. Rev Ciência Saúde 2015;8(3):146-55. https://doi.org/10.15448/1983-652x.2015.3.21360 
6. Laporte GA, Leonardi DF. Transtorno de estresse pós-traumático em pacientes com sequelas de queimaduras. Rev Bras Queimaduras 2010;9(3):105-14.

7. Hochenberry MJ, Wilson D. Wong - Fundamentos de Enfermagem Pediátrica. $9^{\circ}$ ed. São Paulo: Thomson Digital; 2013.

8. Vieira LCV, Fernandes AC, Demitto OM, Bercini OL, Scochi JM, Marcon SS.

Puericultura na atenção primária à saúde: Atuação do enfermeiro. Rev Cogitare Enferm 2012;17(1):119-25. https://doi.org/10.5380/ce.v17i1.26384

9. Fernandes AFMF, Torquato BMI, Dantas ASM, Junior PCAF, Ferreira AJ, Collet N. Queimaduras em crianças e adolescentes: caracterização clínica e epidemiológica. Rev Gaúcha Enferm 2012;33(4):133-41. https://doi.org/10.1590/s198314472012000400017

10. Silva MLR, Junior SAR, Lima LG, Cintra BB, Borges SK. Características epidemiológicas das crianças vítimas de queimaduras atendidas no Hospital de Urgências de Sergipe. Rev Bras Queimaduras 2016;15(3):158-63.

11. Brasil. Ministério da Saúde, Saúde da Criança: Crescimento e Desenvolvimento, Cadernos de Atenção Básica, № 33. Brasília: Ministério da Saúde; 2012.

12. Francisconi GHM, Itakussu YE, Valenciano JP, Fujisawa SD, Trelha SC. Perfil epidemiológico das crianças com queimaduras hospitalizadas em um Centro de Tratamento de Queimados. Rev Bras Queimaduras 2016;15(3):137-41.

13. Viana PF, Resende MS, Tolêdo CM, Silva CR. Aspectos epidemiológicos das crianças com queimaduras internadas no Pronto Socorro para Queimaduras de Goiânia- Goiás. Rev Eletr Enferm 2009;11(4):779-84.

14. Martins GBC, Andrade MS. Queimaduras em crianças e adolescentes: análise da morbidade hospitalar e mortalidade. Acta Paul Enferm 2007;20(4):469-9. https://doi.org/10.1590/s0103-21002007000400013

15. Moraes SP, Ferrari PAR, Sant'anna LF, Raniero WMTJ, Lima SL, Santos MT, Tacla MGTM. Perfil das internações de crianças em um centro de tratamento para queimados. Rev Eletr Enferm 2014;16(3):598-603.

16. Biscegli ST, Benati DL, Faria SR, Boeira RT, Cid BF, Gonzaga TAR. Perfil de crianças e adolescentes internados em Unidade de Tratamento de Queimados do interior do estado de São Paulo. Rev Paul Pediatr 2014;32(3):177-82. https://doi.org/10.1590/0103-0582201432305

17. Millan SL, Gemperli R, Tovo MF, Mendaçolli JT, Gomes SD, Ferreira CM. Estudo epidemiológico de queimaduras em crianças atendidas em hospital de terciário na cidade de São Paulo. Rev Bras Cir Plást 2012;27(4):611-5. https://doi.org/10.1590/s1983-51752012000400024

18. Oliveira DS, Leonardi DF. Sequelas físicas em pacientes pediátricos que sofreram queimaduras. Rev Bras Queimaduras 2012;11(4):234-9.

19. Santos TP, Sá SMP. Ocorrências de queimaduras em crianças em um centro de referência. Revista Baiana de Saúde Publica 2014;38(3):524-38. https://doi.org/10.5327/z0100-0233-2014380300003

20. Pedro ICS, Rinaldi ML, Pan R, Gonçalves N, Rossi LA, Junior JAF, Nascimento LC. Hospitalizações para o tratamento agudo de crianças e adolescentes queimados 20052010. Rev Bras Queimaduras 2014;13(3):154-60.

21. Meschial WC, Sales CCF, Oliveira MLF. Fatores de risco e medidas de prevenção das queimaduras infantis: revisão integrativa da literatura. Rev Bras Queimaduras 2016;15(4):267-73.

22. Takino MA, Valenciano MJ, Itakussu EY, Kaktsuka EE, Fujisawa DS. Perfil epidemiológico de crianças e adolescentes vítimas de queimaduras admitidos em um centro de tratamento de queimados. Rev Bras Queimaduras 2016;15(2):74-9. 